University of Nebraska - Lincoln

DigitalCommons@University of Nebraska - Lincoln

$4-15-2006$

\title{
Magnetic reversal in three-dimensional exchange-spring permanent magnets
}

Jeffrey E. Shield

University of Nebraska - Lincoln, jshield@unl.edu

J. Zhou

University of Nebraska - Lincoln

S. Aich

University of Nebraska - Lincoln

V.K. Ravindran

University of Nebraska - Lincoln

Ralph Skomski

University of Nebraska-Lincoln, rskomski2@unl.edu

See next page for additional authors

Follow this and additional works at: https://digitalcommons.unl.edu/physicssellmyer

Part of the Physics Commons

Shield, Jeffrey E.; Zhou, J.; Aich, S.; Ravindran, V.K.; Skomski, Ralph; and Sellmyer, David J., "Magnetic reversal in three-dimensional exchange-spring permanent magnets" (2006). David Sellmyer Publications. 3.

https://digitalcommons.unl.edu/physicssellmyer/3

This Article is brought to you for free and open access by the Research Papers in Physics and Astronomy at DigitalCommons@University of Nebraska - Lincoln. It has been accepted for inclusion in David Sellmyer Publications by an authorized administrator of DigitalCommons@University of Nebraska - Lincoln. 


\section{Authors}

Jeffrey E. Shield, J. Zhou, S. Aich, V.K. Ravindran, Ralph Skomski, and David J. Sellmyer 


\title{
Magnetic reversal in three-dimensional exchange-spring permanent magnets
}

\author{
J. E. Shield ${ }^{\text {a) }}$ \\ Department of Mechanical Engineering and Center for Materials Research and Analysis, \\ University of Nebraska-Lincoln, Lincoln, Nebraska 68588 \\ J. Zhou \\ Center for Materials Research and Analysis, University of Nebraska-Lincoln, Lincoln, Nebraska 68588
}

S. Aich and V. K. Ravindran

Department of Mechanical Engineering, University of Nebraska-Lincoln, Lincoln, Nebraska 68588

R. Skomski and D. J. Sellmyer

Department of Physics and Astronomy and Center for Materials Research and Analysis, University of Nebraska-Lincoln, Lincoln, Nebraska 68588

(Presented on 31 October 2005; published online 19 April 2006)

\begin{abstract}
In this paper, we investigate the magnetization reversal in single-phase $\mathrm{RE}_{2} \mathrm{Fe}_{14} \mathrm{~B}$ and two-phase $\alpha-\mathrm{Fe} / \mathrm{RE}_{2} \mathrm{Fe}_{14} \mathrm{~B}$ with varying nanoscale grain structures and intergranular exchange interactions produced via controlled segregation during crystallization. We show that the loss of coercivity arises because domain-wall processes dominate the magnetic reversal as the exchange interactions increase. Micromagnetic modeling corroborates a transition to strongly cooperative magnetic reversal as the exchange interactions increase. The magnetic reversal is controlled by the growth of interaction domains via discrete domain-wall motion, and the coercivity is intrinsically limited by the presence of interaction domains. To alleviate this problem, we have built an additional length scale into the structure that is below the interaction domain size but above the limit for intergranular exchange interactions to be significant. These "single-interaction domain" structures retain nucleation-type magnetic reversal and high coercivity. We show experimentally that nanocomposite $\mathrm{Sm}-\mathrm{Co} / \mathrm{Co}$ with this additional length scale has excellent coercivity and nucleation-controlled reversal. (C) 2006 American Institute of Physics. [DOI: 10.1063/1.2163837]
\end{abstract}

\section{INTRODUCTION}

Understanding the magnetic reversal processes is critical when designing microstructures that optimize magnetic performance. In exchange-spring permanent magnets, actual magnetic performance falls far short of the properties predicted for ideal structures. ${ }^{1}$ Much of this discrepancy is due to a lack of overall understanding of the magnetic reversal processes that control the demagnetization of exchangecoupled magnetic systems, making it difficult to design specific structural features that would enable the material to resist demagnetization.

The magnetic reversal processes that occur in exchangespring permanent magnets are complicated by many factors involving structural aspects of the system as well as magnetic interactions. A great deal of understanding of the initiation of magnetic reversal in exchange-coupled systems has been made by studying model bi- and trilayer systems. ${ }^{2-5}$ In strongly exchange-coupled material where the soft phase is completely coupled with the hard magnetic phase, reversal of the hard and soft phase occurs simultaneously. As the dimension of the soft magnetic layer increases, some material away from the interface is not coupled to the hard magnetic layer. In this case, reversal initiates in the soft layer and proceeds in primarily two steps. ${ }^{6-8}$ The first, at low fields, involves an

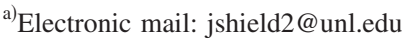

untwisting of the spiral (which resembles a domain wall), while the second, at higher fields, involves an out-of-plane rotation of the soft-phase (usually $\mathrm{Fe}$ ) moments. ${ }^{9}$ In granular, three-dimensionally assembled nanocomposite permanent magnets, reversal initiation in the soft magnetic phase has been observed as well. ${ }^{10}$

Propagation of the magnetic reversal in strongly exchange-coupled systems is not as well understood as the initiation stage. Ideally, individual grains reverse independently from one another. In dilute magnetic systems, this ideal can be approached. ${ }^{11,12}$ At higher volume fraction of magnetic phases, magnetostatic interactions have been shown to lead to more cooperative reversal. ${ }^{13}$ In exchangespring permanent magnets, the addition of exchange interactions leads to additional complications, such as the formation of interaction domains, that influence the magnetic reversal process.

\section{EXPERIMENTAL PROCEDURES}

Several different rare-earth magnetic alloys will be examined here, all produced by melt spinning. Samples of stoichiometric $\mathrm{Nd}_{2} \mathrm{Fe}_{14} \mathrm{~B}$ and Nd-Pr-Dy-Fe-Co-B-Ti-C-Zr (the "nine component," or 9C, sample) are single phase with the 2-14-1 tetragonal structure, ${ }^{14}$ two-phase Pr-Fe-B-Zr nanocomposite of $\alpha$-Fe and 2-14-1, ${ }^{15}$ and two-phase Sm-Co with fcc Co and the $\mathrm{TbCu}_{7}$-type $\mathrm{SmCo}_{7}$ structure. ${ }^{16}$ Details on the 


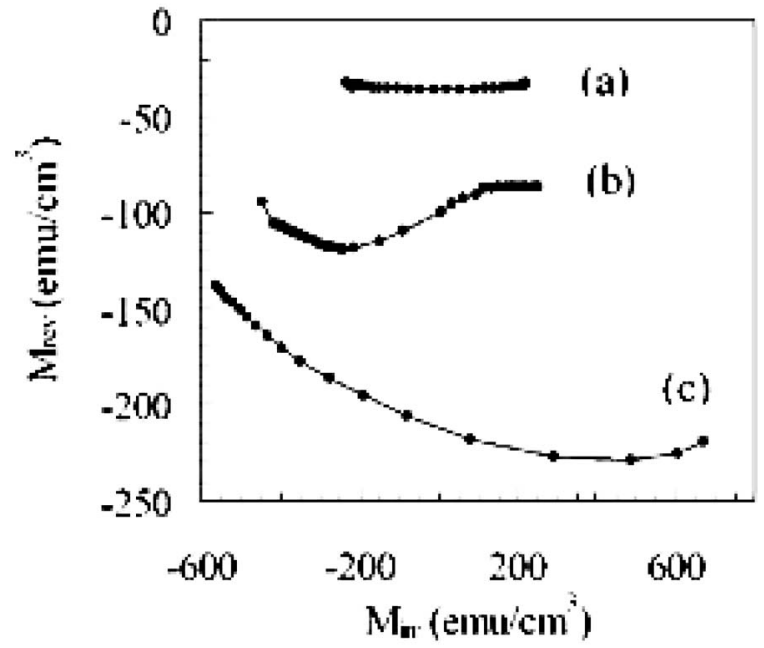

FIG. 1. Reversible magnetization as a function of irreversible magnetization for single-phase (a) Nd-Pr-Dy-Fe-Co-B-Ti-Zr-C and (b) Nd-Fe-B, and for (c) two-phase nanocomposite $\mathrm{Pr}-\mathrm{Fe}-\mathrm{Co}-\mathrm{B}-\mathrm{Nb}$. All curves are at a reverse field of $6 \mathrm{kOe}$.

sample preparation and structural characterization of the various alloys have been presented elsewhere. ${ }^{14-16}$ The detailed microstructural analysis has been reported previously. ${ }^{14}$ Magnetic characterization was accomplished at room temperature using vibrating sample (Lakeshore) or SQUID (Quantum Design MPMS) magnetometers. Samples were magnetized either using a $3.5 \mathrm{~T}$ pulse magnetizer or in a $5 \mathrm{~T}$ magnetic field. Recoil measurements using the dc demagnetization (DCD) technique and data analysis outlined by Crew and Lewis ${ }^{17}$ were also conducted. Modeling studies utilizing object oriented micromagnetic framework (OOMMF) were conducted on isotropic single-phase grain structures with a grain size of $60 \mathrm{~nm}$.

\section{RESULTS AND DISCUSSION}

The as-solidified stoichiometric Nd-Fe-B-based alloys were amorphous and were crystallized $5 \mathrm{~K}$ above their respective crystallization temperatures. ${ }^{18} \mathrm{X}$-ray diffraction revealed only the 2-14-1 tetragonal phase for both alloys. Grain sizes were measured to be $77 \pm 30 \mathrm{~nm}$ and $60 \pm 13 \mathrm{~nm}$ for the ternary and 9C samples, respectively. During crystallization, the alloying elements, notably $\mathrm{Ti}$ and $\mathrm{C}$, were expelled to the grain-boundary regions. More complete microstructural characterization of these alloys has been presented elsewhere. ${ }^{14}$ At higher heat treatment temperatures, the Ti and $\mathrm{C}$ form $\mathrm{TiC}$ precipitates. ${ }^{19}$ The segregation of $\mathrm{Ti}$ and $\mathrm{C}$ significantly influenced the magnetic behavior of the material. The ternary alloy had a coercivity of approximately 3 $\mathrm{kOe}$, while the $9 \mathrm{C}$ alloy had a coercivity of $12 \mathrm{kOe}$. As the difference in grain size was minimal, the significantly lower coercivity for the ternary alloy is primarily the result of different reversal mechanisms, as revealed through the DCD measurements. Figure 1 shows the relationship between the reversible $\left(M_{\text {rev }}\right)$ and irreversible $\left(M_{\text {irr }}\right)$ components of the magnetization at $6 \mathrm{kOe}$. A linear or flat relationship suggests nucleation-controlled reversal, while the development of concavity suggests that domain processes are becoming increasingly important in the reversal processes. ${ }^{17}$ The curve for the $9 \mathrm{C}$ alloy is nearly linear with very little variation in $M_{\text {rev }}$ over the extent of $M_{\text {irr }}$. This is contrasted with the ternary alloy, which is not linear and varies extensively over $M_{\text {irr. }}$ This behavior suggests that domain-wall processes are contributing to the demagnetization process. The different behavior is attributed to the isolation of the grains by the segregating $\mathrm{Ti}$ and $\mathrm{C}$. The $\mathrm{Ti}$ and $\mathrm{C}$ at the grain boundaries inhibit exchange interactions, leading to less cooperative magnetic reversal. The Pr-Fe-B nanocomposite, with a grain size of $17.8 \mathrm{~nm}$, coercivity of $7 \mathrm{kOe}$, and energy product of 16.1 MGOe ${ }^{14}$ displayed behavior similar to the ternary NdFe-B single-phase alloy. The $M_{\text {rev }}$ vs $M_{\text {irr }}$ curve was concave, suggesting pinning-dominated reversal [Fig. 1(c)].

Even though the grain sizes of the alloys are well below the single domain limit, and the grains are substantially defect free, the $M_{\text {rev }}$ vs $M_{\text {irr }}$ curves suggest first that domain processes are active and second that the domain walls are pinned. The domain-wall processes in these materials originate from interaction domains that exist due to strong intergranular interactions. The interaction domains allow cooperative reversal, as reversal proceeds grain by grain, i.e., interaction domains favorably aligned with the applied magnetic field grow. Micromagnetic calculations on model $\mathrm{Nd}_{2} \mathrm{Fe}_{14} \mathrm{~B}$ systems with strong and weak interactions reveal that, with strong interactions, magnetic reversal becomes incoherent, suggesting that more discrete domain-wall processes are occurring. These modeling studies corroborate the experimental findings that stronger intergranular exchange interactions lead to magnetization reversal processes that are more cooperative, proceeding grain-by-grain rather than independently.

In the promising two-phase exchange-spring permanent magnets, a dramatic loss of coercivity as the soft magnetic phase fraction increases and the grain size decreases has limited the energy densities that have been achieved. This loss of coercivity, as we have shown, is directly related to the strong exchange interactions through the development of interaction domains and subsequent domain-wall reversal modes. However, the strong intergranular interactions also result in enhanced remanence values and high-energy products, aspects of these materials that originally attracted attention. Thus it is now the challenge to the materials engineer to develop microstructures that better resist demagnetization while retaining high remanence and energy products. One approach would be to develop materials that better inhibit domain-wall motion and the growth of interaction domains. One difficulty with this approach is that altering the grain boundaries likely will also have detrimental effects on the intergranular exchange interactions, as was observed here with the segregation of $\mathrm{Ti}$ and $\mathrm{C}$. Compositionally graded grain boundaries may enhance exchange coupling and improve pinning, as has been observed in multilayered materials, ${ }^{20,21}$ but are practically difficult to achieve using bulk processing routes.

Another route may be to eliminate the presence of interaction domains altogether by, for example, developing an additional length scale in the structure. The interaction domain size scales with the intrinsic magnetic properties just as the single domain limit does and is proportional to 


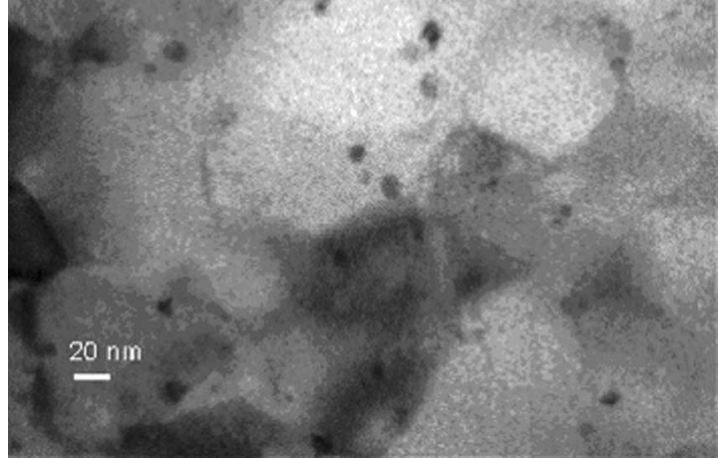

FIG. 2. Transmission electron micrograph of a rapidly solidified $\mathrm{Sm}-\mathrm{Co}$ alloy showing soft magnetic fcc Co precipitates imbedded in a hard magnetic $\mathrm{SmCo}_{7}$ grains. The $\mathrm{SmCo}_{7}$ grains are below the single domain limit but above the size necessary for the exchange-spring effect.

$(A / K)^{1 / 2} \cdot{ }^{22}$ Two possibilities exist here: first, one could generate particles on the order of hundreds of nanometers, which is typically the order for single domain particles of hard magnetic materials. The particles would then contain a nanoscale substructure of hard and soft magnetic phases. The difficulty here is in the fabrication of $100-500 \mathrm{~nm}$ particles. In the second scenario, the grain size of the hard magnetic phase is on the order of hundreds of nanometers and has nanoscale soft magnetic grains imbedded in it. This structure has been successfully fabricated in rapidly solidified $\mathrm{Sm}-\mathrm{Co}$ alloys (Fig. 2). ${ }^{16}$ The structure consists of hard magnetic $\mathrm{SmCo}_{7}$ grains on the order of a few hundred nanometers. Intragranular Co precipitates on the order of $10 \mathrm{~nm}$ provide the soft magnetic component, and the fine scale ensures excellent coupling to the surrounding hard magnetic phase. ${ }^{16}$ This material was shown to have high remanence ratios despite its isotropic grain structure with $\sim 150 \mathrm{~nm} \mathrm{SmCo}_{7}$ grains, but maintained a coercivity of almost $10 \mathrm{kOe}$ in the as-solidified condition. ${ }^{16}$ Because of the larger-scale $\mathrm{SmCo}_{7}$ grains, intergranular interactions that lead to the development of interaction domain were minimal. Figure 3 shows the relationship between $M_{\text {rev }}$ and $M_{\text {irr }}$ for this sample. It shows a short "hook" followed by a nearly linear region. The hook suggests the nucleation of a reverse domain, which then grows with very little resistance, essentially nucleationcontrolled magnetic reversal. Initial magnetization curves of this alloy also indicate that nucleation events control the magnetization process. ${ }^{23}$ Further alloy and process development will undoubtedly uncover other systems with outstanding magnetic performance.

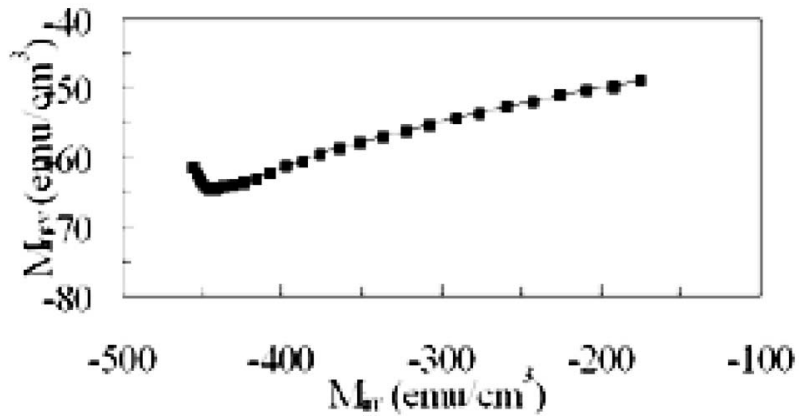

FIG. 3. Reversible magnetization $\left(M_{\text {rev }}\right)$ as a function of irreversible magnetization $\left(M_{\text {irr }}\right)$ for the Sm-Co alloy.

\section{CONCLUSIONS}

In this paper, we have shown that the magnetic reversal processes in exchange-spring permanent magnets are dominated by domain-wall processes. The presence of interaction domains is largely responsible for the domain-controlled demagnetization behavior. We have also proposed grain structures that can effectively eliminate interaction domains by generating an additional length scale larger than the nanoscale but below the single domain limit. A model system with $\sim 150 \mathrm{~nm} \mathrm{SmCo}_{7}$ grains with intragranular Co precipitates and exchange-spring characteristics has been shown to have nucleation-controlled magnetization processes. As a result, improved materials can be developed that will approach theoretical predictions for exchange-spring nanocomposite permanent magnets.

The authors appreciate useful discussions with D.C. Crew, and sample preparation from B. Kappes and D.J. Branagan. The compositional profiles were obtained through the SHaRE program at Oak Ridge National Laboratory, and assistance from $\mathrm{J}$. Bentley is greatly appreciated. This project was supported by the NSF-MRSEC QSPINS, the Nebraska Research Initiative, the Center for Materials Research and Analysis, the National Science Foundation through grant No. DMR0305354, and the Department of Energy.

${ }^{1}$ R. Skomski and J. M. D. Coey, Phys. Rev. B 48, 15812 (1993).

${ }^{2}$ I. A. Al-Omari and D. J. Sellmyer, Phys. Rev. B 52, 3441 (1995).

${ }^{3}$ M. Shindo, M. Ishizone, H. Kato, T. Miyazaki, and A. Sakuma, J. Magn. Magn. Mater. 161, 1 (1996)

${ }^{4}$ J. S. Jiang, E. E. Fullerton, J. M. Grimsditch, C. G. Sowers, J. Pearson, and S. D. Bader, Philos. Mag. B 80, 247 (2000).

${ }^{5}$ J. S. Jiang and S. D. Bader, Mat. Sci. Technol. 17, 1491 (2001).

${ }^{6}$ E. Goto, N. Hayashi, T. Miyashita, and K. Nakagawa, J. Appl. Phys. 36, 2951 (1965).

${ }^{7}$ E. E. Fullerton, J. S. Jiang, and S. D. Bader, J. Magn. Magn. Mater. 200, 392 (1999).

${ }^{8}$ E. E. Fullerton, J. S. Jiang, M. Grimsditch, C. H. Sowers, and S. D. Bader, Phys. Rev. B 58, 12193 (1998).

${ }^{9}$ C. L. Platt, A. E. Berkowitz, S. David, E. E. Fullerton, J. S. Jiang, and S. D. Bader, Appl. Phys. Lett. 79, 3992 (2001).

${ }^{10}$ Y. Gao, D. Shindo, and A. K. Petford-Long, J. Appl. Phys. 93, 8119 (2003).

${ }^{11}$ Er. Girt, Kannan M. Krishnan, G. Thomas, and Z. Altounian, Appl. Phys. Lett. 76, 1746 (2000).

${ }^{12}$ Yingfan Xu, M. L. Yan, J. Zhou, and D. J. Sellmyer, J. Appl. Phys. 97, 10J320 (2005).

${ }^{13}$ D. C. Crew, Er. Girt, D. Suess, T. Schrefl, K. M. Krishnan, G. Thomas, and M. Guilot, Phys. Rev. B 66, 184418 (2002).

${ }^{14}$ J. E. Shield, B. B. Kappes, D. J. Branagan, and J. Bentley, J. Magn. Magn. Mater. 246, 73 (2002).

${ }^{15}$ J. E. Shield, Y. Liu, R. Marr, Z. Chen, and B. M. Ma, IEEE Trans. Magn. 40, 2901 (2004).

${ }^{16}$ J. E. Shield, S. Aich, V. K. Ravindran, A. Hsaio, and L. H. Lewis, Scr. Mater. 52, 758 (2004).

${ }^{17}$ D. C. Crew and L. H. Lewis, J. Appl. Phys. 87, 4783 (2000).

${ }^{18}$ B. B. Kappes and J. E. Shield, J. Non-Cryst. Solids 317, 106 (2003).

${ }^{19}$ D. J. Branagan and R. W. McCallum, J. Alloys Compd. 230, 67 (1995).

${ }^{20}$ D. C. Crew, L. H. Lewis, J. Kim, and K. Barmak, J. Appl. Phys. 89, 7528 (2001).

${ }^{21}$ J. S. Jiang, J. E. Pearson, Z. Y. Liu, B. Kabius, S. Trasobares, D. J. Miller, S. D. Bader, D. R. Lee, D. Haskel, G. Srajer, and J. P. Liu, J. Appl. Phys. 97, 10K311 (2005).

${ }^{22}$ K. Amaravadi, M.S. thesis, University of Nebraska-Lincoln, 2004.

${ }^{23}$ S. Aich, J. E. Shield, A. Hsaio, and L. H. Lewis, IEEE Trans. Magn. 40, 2913 (2004). 